\title{
Quantum optics laboratories for teaching quantum physics
}

\section{Enrique Galvez}

Enrique J. Galvez, "Quantum optics laboratories for teaching quantum physics," Proc. SPIE 11143, Fifteenth Conference on Education and Training in Optics and Photonics: ETOP 2019, 111431A (2 July 2019); doi:

$10.1117 / 12.2523843$

SPIE Event: Fifteenth Conference on Education and Training in Optics and Photonics: ETOP 2019, 2019, Quebec City, Quebec, Canada 


\title{
Quantum Optics Laboratories for Teaching Quantum Physics
}

\author{
Enrique J. Galvez \\ Department of Physics and Astronomy, Colgate University, Hamilton, New York 13346, U.S.A.
}

\begin{abstract}
With the coming of quantum information and associated technologies, there is increasing need to prepare students on the fundamentals of quantum physics. Fundamental physical principles can be hidden by the mathematical formalism of quantum mechanics. Thus it is necessary to expose students to experiments that illustrate fundamentals. Quantum optics experiments based on the generation of twin photons via spontaneous parametric down-conversion have many advantages, such as being simple, reliable, and of reasonable cost. These types of experiments are ideal for the advanced laboratory in the physics curriculum. At Colgate University we offer them as a laboratory package that is associated with our upper-level undergraduate course on quantum mechanics. In this article we present our latest approaches. Among them is a new layout for the experiments to provide a more intuitive physical picture.
\end{abstract}

Keywords: Quantum optics, Single photons, Undergraduates, Quantum mechanics

\section{INTRODUCTION}

Quantum information constitutes a field that promises many scientific and technological advances. It exploits aspects of quantum physics that were once considered academic, but which are now central to applications. Among these fundamental effects, quantum entanglement, stands as a key piece in the development of quantum computers. In communications, quantum key distribution, a technology that uses quantum principles to implement an effective method of communication security, is already being deployed commercially. Thus, quantum technologies may soon be part of our everyday life. Yet for all these advances, progress in educating the workforce that develops and implements these technologies has been slow. The efforts in education have to involve new teaching methodologies, new textbooks, and new teaching laboratories. The latter activity is where we devote our efforts, and our latest updates on them are described in this article.

The laboratory setting for teaching fundamentals of quantum physics uses light. It is an unusual source. Not a laser, but a source derived from a laser beam. The source entails the generation of photon pairs via the nonlinear process of spontaneous parametric down-conversion. These photon pairs have unique properties: they are produced simultaneously, and are entangled in energy and momentum. As a consequence, they are correlated in their direction of propagation, and can constitute a recreation of the famous thought experiment of Einstein, Podolski and Rosen (EPR). ${ }^{1,2}$ Minor modifications allow one to obtain polarization entangled photons. ${ }^{3}$ The correlations between these polarization-entangled photons have been used many times for measurements of violations of Bell inequalities to ever more stringent conditions, ${ }^{4}$ showing that nature is indeed non-local and non-realistic.

It has only been 17 years since the first implementation of entanglement and Bell inequalities in the undergraduate laboratory. ${ }^{5}$ Since then numerous other implementations have been reported, which focus on other fundamental aspects of quantum physics. ${ }^{6}$ Other variations of Bell implementations have also been reported..$^{7,8}$ An important experiment in this suite of teaching demonstrations is the Hanbury-Brown and Twiss experiment. ${ }^{9,10}$ This experiment focuses on demonstrating the wholeness of a photon, by sending single photons to a beam splitter and measuring anticorrelations in the reflection and transmission. The quantum eraser is an experiment with an interferometer where a single photon interferes with itself or not depending on whether the paths of the interferometer are indistinguishable or indistinguishable, respectively. ${ }^{11,12}$ The key point in the quantum eraser is that the decision of whether interference should be observed or not is done after the light has passed

Further author information: E.J.G.: E-mail: egalvez@colgate.edu (Send correspondence to E.J.G.)

E.J.G.: E-mail: egalvez@colgate.edu, Telephone: 1315 228-7205

Fifteenth Conference on Education and Training in Optics and Photonics: ETOP 2019, edited by

Anne-Sophie Poulin-Girard, Joseph A. Shaw, Proc. of SPIE Vol. 11143, 111431A · (c) 2019

SPIE, ICO, IEEE, OSA · CCC code: 0277-786X/19/\$18 · doi: 10.1117/12.2523843

Proc. of SPIE Vol. $11143111431 \mathrm{~A}-1$ 
the interferometer. An extension of this line of though in this type experiment is known as delayed choice, where the decision is made no only after the light has gone through the interferometer, but after it has been detected. One implementation of this involves the delayed use of polarization measurements on the polarization-entangled partner, ${ }^{13}$ and a more recent one involves delayed filtering on an energy-entangled partner. ${ }^{14}$ Finally, there are interesting outcomes when both photons act as one, also known as the biphoton, and interfere due to the indistinguishability in when they were created. ${ }^{15}$ One of the most important experiments in quantum optics that is the basis of many scientific and technological implementations is the Hong-Ou-Mandel experiment. Its implementation in a quantum optics teaching laboratory has been reported for non-collinear ${ }^{16}$ and collinear cases. ${ }^{17}$ We have recently completed a demonstration of a quantum application connected to quantum key distribution, and expect to report on it in the near future.

Current laboratories mostly use a V-type of layout for non-collinear down-conversion, where photon pairs come out at a shallow angle (typically 3 degrees) from the crystal that generates them. Thus, photon paths are next to each other. In the present manuscript I report on a new T-type layout where photons are still emerging from the crystal at shallow angles, but are steered to take opposite paths before they reach optical elements in a given experiment. The two layouts are shown in Fig. 1.

(a)

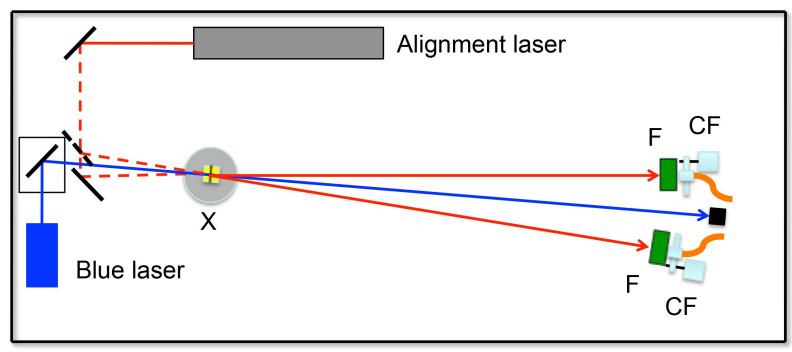

(b)

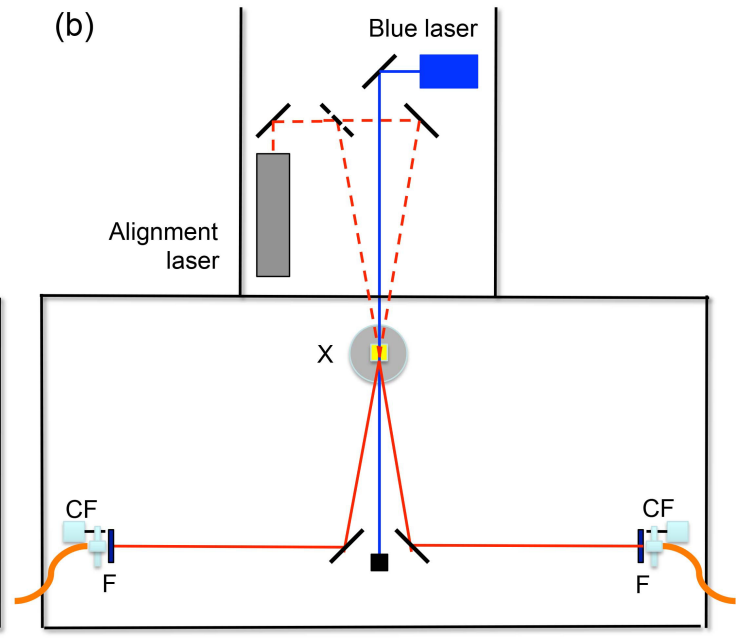

Figure 1. Schematic of two layouts of the apparatus (a) the traditional "V" shaped and (b) the new "T" shape that we have investigated and implemented. Basic optical components are the down-conversion crystal (X), bandpass filters (F) and collimator-fiber assembly $(\mathrm{CF})$ to collect the photons into multimode fibers to send them to detectors.

\section{LABORATORIES}

\subsection{Components}

The apparatus has a few basic components that we discuss in detail. We have more information in our website. ${ }^{18}$

\subsubsection{The Pump laser}

The source of power in the experiments is an input laser beam, also called the pump. Spontaneous parametric down-conversion involves converting one photon into two. From conservation of energy, the down-converted photons have half the energy and thus twice the wavelength of the incident photon. Thus we have to worry about a light source producing photons a certain wavelength and detectors detecting photons at twice the wavelength. The most convenient compromise between a reasonably priced short-wavelength laser and efficient detectors leads us to the gallium-nitride $(\mathrm{GaN})$ diode lasers (the same kind used in blu-ray players), with a wavelength of $405 \mathrm{~nm}$. These lasers have become so inexpensive that one can get them for as low as $\$ 20$ (as laser pointers). More reliable lasers from laser companies offer them at $\$ 300-\$ 1000$, with current-control stability. The power of the laser should be in the range $20-50 \mathrm{~mW}$. Higher powers are available, but they are overkill 
and hazardous for students to handle. Some companies offer GaN lasers with greater stability and temperature control for $\$ 6000-\$ 7000$, although in general all experiments can be done with the inexpensive lasers. There are a number of vendors, so we do not recommend any one in particular.

\subsubsection{The Down-Conversion Crystal}

The crystal of choice for the experiments is a solid block $(5-\mathrm{mm} \times 5-\mathrm{mm} \times 3-\mathrm{mm})$ of beta barium borate to produce type-I down-conversion. The crystal choice and the type-I down-conversion makes it very simple to use. We will not discuss the details of the process, as has been done before. ${ }^{11}$ The main idea is that the crystal is birefringent and is set up for down-conversion by having the crystal axis in one plane (say horizontal) and forming a certain (phase-matching) angle with the incoming beam direction. The pump beam also has its polarization in the same plane (horizontal). The emission of photon pairs depends on the phase-matching angle that the crystal axis forms with the polarization of the pump beam. For the wavelength of the laser that we use and for generating pairs in a 3-degree angle, this angle is $29^{\circ}$. There is a vendor that has this item on stock (Newlight Photonics, part number NCBBO5300-405(I)-HA3, \$600). The crystal in this arrangement produces pairs of photons that are vertically polarized.

For making polarization-entangled photons we use a system of two thin crystals rotated by 90 degrees. When the input beam is polarized 45 degrees to the horizontal, the horizontal component produces vertically polarized pairs in one crystal and the vertical component produces horizontally-polarized pairs in the other crystal. The crystals are thin enough that is indistinguishable which crystal is the source of the pairs, and so they are in a non-separable or entangled state:

$$
|\psi\rangle=\frac{1}{\sqrt{2}}\left(|H\rangle_{1}|H\rangle_{2}+|V\rangle_{1}|V\rangle_{2} e^{i \delta}\right)
$$

These crystals are also sold as a package and mounted, with the proper antireflection coatings (Newlight Photonics, part number PABBO5050-405(I)-HA3, \$1000). The desired state is obtained by setting $\delta=0$ with a 6-mm thick quartz crystal (Newlight Photonics, part number QAR25550-A-AR405, \$430).

\subsubsection{Detectors}

One of the factors determining the light being used in the experiments is the efficiency of the detectors of single photons. The ones that are most available have an efficiency that peaks around $700 \mathrm{~nm}$. At twice the wavelength of the GaN diode laser, $810 \mathrm{~nm}$, the efficiency of the detectors is about $60 \%$, which is reasonable good. The best option for these detectors are Excelitas' educational modules (part number SPCM-EDU CD3375, \$1700 each), acquired through the Advanced Laboratory Physics Association (ALPHA) ${ }^{19}$ These detectors are fiber coupled, so we use fiber collimators (a lens system, Thorlabs, part number F220FCB, \$145) that focuses the light into a multimode fiber (Thorlabs, part number M31L01 \$50). These components are labeled "CF" in Fig. 1.

Photomultipliers are not efficient at $810 \mathrm{~nm}$, and so they are not suitable. In the experiments we record the coincidences so the signal depends on the product of the detector efficiencies.

\subsubsection{Electronics}

In the experiments we need to record the signals from the two photons. The detectors put out digital pulses recording the arrival of photons. We record the arrival of a pair of photons using coincidence circuits. One can use pulse-electronic modules, but the arrival of smart electronics such as field programmable gate array (FPGA) has made this effort much easier, as one only needs to feed the pulses to the FPGA board and program it to record and count the coincidences. These devices are easily interfaced with a computer. The Altera DE2 educational board $(\$ 350)$ is an economical and versatile circuit that has been adapted to these experiments. ${ }^{20}$ There are other options, such as Red Dog Physics model CD48 (\$250).

\subsubsection{Alignment Laser}

The photon pairs constitute a very weak stream of photons, so we cannot see them reflected off a card or screen. Yet we need to align the optics to steer the photons through the apparatus and then into fibers that send those photons to the detectors. We accomplish this alignment with an auxiliary laser beam that follows the path of the down-converted photons. This laser can be a helium-neon (HeNe), our preference, but it can be another laser beam with a circular gaussian profile. Hand-held fiber lasers can be a convenient and less expensive alternative. 


\subsubsection{Other Components}

The V-type design fits in a 2 -ft $\times 5$ - $\mathrm{ft} \times 4.3$ in breadboard (Thorlabs, part number B2460G, \$1700) or metric equivalent $750-\mathrm{mm} \times 1500-\mathrm{mm} \times 110-\mathrm{mm}$ (Thorlabs, part number B75150B, \$1700). The "T" type needs an additional breadboard attached to the one mentioned above. Alternatively, the "T" design is easily adapted in an optical table, if it is available.

Other components, such as mirrors and mounts are standard. Which ones to get depends on the particular experiment. However, 15 years of experience with these laboratories has led us to make some specific recommendations:

- Rotational Mounts. Most optical experiments will involve waveplates, crystals, polarizers, which use rotational mounts. These mounts are useful when their zero reading matches the axis of the optical component. There are two types of rotational mounts that allow you to reset the zero after calibration. The best in our view are from Optosigma, part number PH-25.4-ARS-UU, \$170; but the other alternative is good as well: Thorlabs, part number RSP1x15, $\$ 130$.

- Mounts. The optical elements have to be laid out so that the beam is as close to the breadboard as possible (3 in or $7.5 \mathrm{~cm}$ ). For interferometers we recommend mounting the components on pedestal mounts for stability. Mounts for mirrors and collimators can be magnetic mounts, which are convenient to attach to the table. The square mounts are also important for use in conjunction with the curved surfaces used in the alignment, described below. There are numerous vendors for these options, so we do not recommend any one in particular.

- Polarizers. There are no good film polarizers for $810 \mathrm{~nm}$. The best available are calcite Thompson prisms, which are good throughout the visible and have high extinction ratios.

- Waveplates. Numerous experiments require waveplates. We recommend zero-order waveplates at the standard wavelength of $808 \mathrm{~nm}$.

- Band-pass filters, The collimators must be preceded by bandpass filters, which block many non-downconversion photons. A typical bandwidth ranges between $10 \mathrm{~nm}$ and $40 \mathrm{~nm}$. Least expensive filters come at predetermined center wavelengths. Therefore, early in the process of purchasing equipment, one must make sure that the pairs of equal energy can pass through the filters. Because of variations in the wavelength of the pump laser that one can obtain, it is possible to get filters that are not appropriate.

- Interferometer optics We use Mach-Zehnder type interferometers. For best results the beams splitters and mirrors have to be identical, bought together. This insures that their reflection coefficients are about the same, producing the largest fringe visibilities.

\section{THE T-TYPE LAYOUT}

The layout of Fig. 1(a) is compact, fits in an optical breadboard, and has been proven to be reliable and effective. It has one cosmetic weakness: photon pairs, with all of their marvelous quantum properties travel side by side. Their nonlocal properties tied to entanglement remain the same regardless of the paths they take. Thus, it could be pedagogical to emphasize this property of the state of the photons by sending them in opposite directions.

We currently offer a semester-long laboratory on photon experiments tied to an upper-level undergraduate course on quantum mechanics. There are a number of textbooks available today that can be used with this

laboratory. ${ }^{21-23}$ In the labs, the students set up the experiments in one- or two-week sessions, to then do the experiment the following week. We have successfully implemented this approach in 2018. In 2019 we decided to try the "T" layout of Fig. 1(b). Key aspects of the alignment are shown in Fig. 2

The first step in the alignments is to set up the path that the light has to follow. The dimensions shown in Fig. 2(a) are for generating pairs at $\pm 3^{\circ}$ and for placing the fiber collimator at about 1-m away from the crystal. Following the labels in Fig. 2, step (1) involves making the pump beam follow a straight trajectory parallel to the holes of the breadboard, and in doing so defining the axis of the down-conversion process. Step (2) involves 

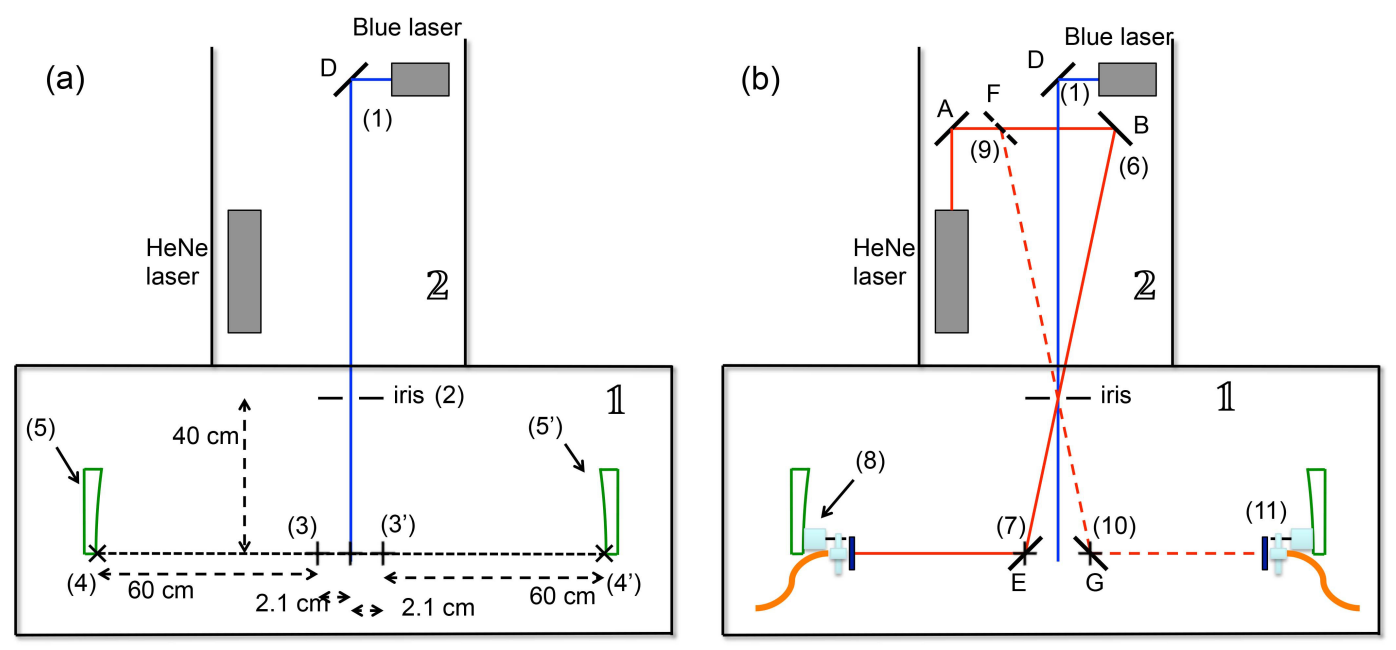

Figure 2. Schematic of steps followed in setting up the experiments. In (a) we give the dimensions used in the experiments. In (b) we show the layout for the alignment of the optical components.

putting an iris in the location where the down-conversion crystal will be placed. The next step is to mark the breadboard at points that define the path of the down-converted photons. This includes a place where they are steered (3) and $\left(3^{\prime}\right)$, and their final destination, points (4) and $\left(4^{\prime}\right)$. The segments from (3) to (4), and $\left(3^{\prime}\right)$ to $\left(4^{\prime}\right)$, define trajectories that must be parallel to the holes on the breadboard. Once those points are defined, we place two 3-D printed surfaces (5) and $\left(5^{\prime}\right)$ that have a radius of curvature of $1 \mathrm{~m}$. They must be placed such that the tangents to the surfaces at points $(4)$ and $\left(4^{\prime}\right)$ are perpendicular to the photon trajectories. Points (4) and $\left(4^{\prime}\right)$ are 1-m, away from the iris defining the position of the crystal. The curved surfaces are for alignment corrections done later.

A second step in the alignment involves placing optical components so that the beam from an alignment laser follows the path of the down-converted photons. This is shown in Fig. 2(b). The light from a HeNe laser is steered by mirror A. Placement of mirrors B (6) and E (7) send the light to point (4). This alignment is done with a small plumb bob held by a thread and by looking at the shadow of the thread cast on a screen. Once the beam is aligned we place the fiber collimator (8) with its square mount touching the curved surface. This procedure is then repeated for the other arm. To be able to flip from one beam to the other, mirror $\mathrm{F}$ is flippable. Thus by placing it (9) and mirror G (10) we get the second beam aligned and sent into a fiber collimator (11).

Final steps involve placing the crystal in the location of the iris and then looking for down-conversion photons with the detectors. After turning off the lights of the room and turning the detectors on, the crystal is tilted about a vertical axis until down-conversion detections are obtained. If the signal from the detectors do no peak at the same orientation of the crystal, then we set the crystal for a maximum in one detector and move the collimator for the other detector along the curve surface until it reaches a maximum. This should also maximize the coincidence signal.

\section{LABORATORIES}

The students did 5 experiments in the 14 weeks of the academic semester: polarization Stern-Gerlach, singlephoton interference, the Hanbury-Brown and Twiss experiment, the quantum eraser, delayed choice, entanglement and Bell inequality measurements. Figure 3 shows two experiments that relied on the entanglement between the photons, so that the layout made a particular impact to emphasize that the photon correlations are independent of where the photons are and in what direction they are traveling, as shown here.

In Fig. 3(a) we show the setup for the delayed-choice experiment. The photon on the left goes through a long optical fiber to then pass through a filter before going to the Alice detector. The filter decides whether we should see interference when the other photon goes through the interferometer and is received by Bob. The delayed 
choice aspect of the experiment is implemented by the fiber delay, because the passage though the filter at Alice occurs after the photon reaching Bob has already been detected. To receive the signals from both photons at the same time by the coincidence unit, the electronic signal from Bob is delayed by an electric cable, as shown in the figure.

In an early lab on the Hanbury-Brown and Twiss experiment students got an anti-correlation parameter $g_{2}^{(0)}=0.41 \pm 0.01$. The objective of this experiment is to test whether single photons act like a wave or a particle when they pass through a beam splitter. A wave would give $g_{2}^{(0)}=1$ and a particle would give $g_{2}^{(0)}=0$. They did this not by using a beam splitter, but by using the interferometer shown in Fig. 3(a) as the beam splitter and placing a third collimator (Charlie) in the other output port of the interferometer. Optical and electric delays were not used in this experiment. This test completed one of the counter-intuitive aspects of quantum physics, that while going the interferometer the photon acts like a wave showing interference, but when detected it acts like a particle because it is detected in one or the other detectors but not both.

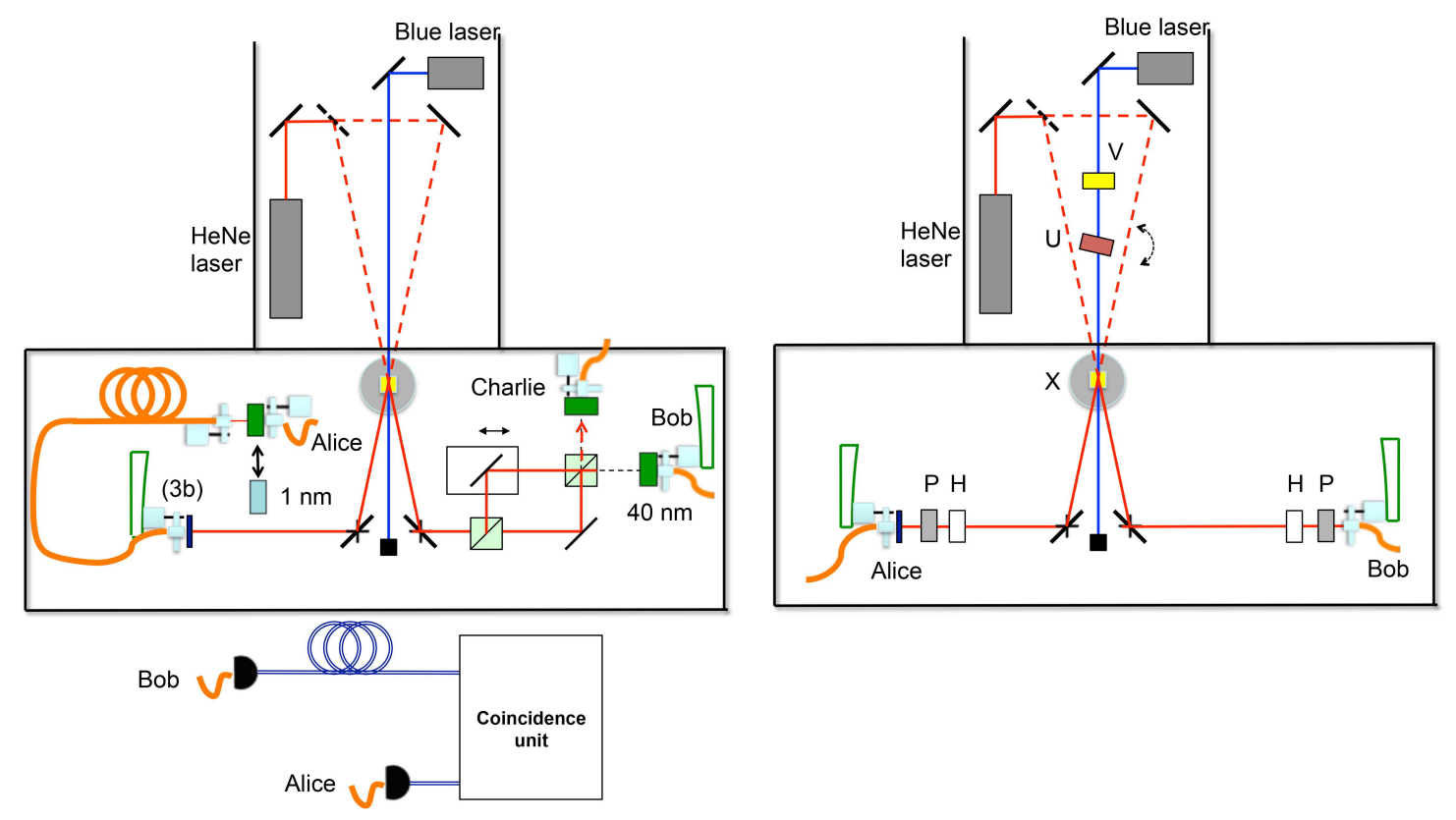

Figure 3. Schematic of the experiments: delayed-choice and Hanbury-Brown and Twiss in (a), and polarization entanglement and Bell inequalities in (b).

In Fig. 3(b) we show the setup for preparing photon pairs in the polarization-entangled states of Eq. 1. In this experiment students study the nonlocal correlations between the photons received by Alice and Bob. ${ }^{15}$ Once they do some tests, they go ahead and take 16 measurements for implementing the Claused-Horne-Shimony-Holt version of a Bell inequality. ${ }^{5}$ Indeed, the students did the corresponding measurements and found $S=2.10 \pm 0.06$. A local realistic understanding of the problem predicts that $S<2$. In addition, the students modified the apparatus to put the photons in a mixed state, where the photons are not in the entangled state, and for that case they got $S=0.12 \pm 0.06$. This is done by introducing a time delay between the polarization states (horizontal-horizontal vs. vertical-vertical) with a 6 - $\mathrm{mm}$ quartz crystal to make the two polarization states distinguishable temporally.

\section{DISCUSSION}

The use of the layout described above was successful in the sense that students got all the expected outcomes of the experiments. The unsuccessful part was that the apparatus was extended over 1-1/2 optical breadboards. It was not compact like in the V-type layout, and so there were more chances for accidents. That is, that a student 
accidentally bumped an an optical component, mostly a mirror, which would ruin most or part of the alignment. Future versions will have to implement a method of also putting barriers to avoid accidents.

\section{ACKNOWLEDGMENTS}

This work was funded by the grant from the National Science Foundation PHY-1506321.

\section{REFERENCES}

[1] Einstein, A., Podolsky, B., and Rosen, N., "Can quantum-mechanical description of physical reality be considered complete?," Phys. Rev. 47, 777-780 (135).

[2] Howell, J., Bennink, R., Bentley, S., and Boyd, R., "Realization of the einstein-podolsky-rosen paradox using momentumand position-entangled photons from spontaneous parametric down conversion," Phys. Rev. Lett. 92, 210403 (2004).

[3] Kwiat, P., Waks, E., White, A., Appelbaum, I., and Eberhard, P., "Ultrabright source of polarizationentangled photons," Phys. Rev. A 60, 773-776 (1999).

[4] Aspect, A., "Closing the door on einstein and bohr's quantum debate," Physics 8, 123 (2015).

[5] Dehlinger, D. and Mitchell, M., "Entangled photons, nonlocality, and bell inequalities in the undergraduate laboratory," Am. J. Phys. 70, 903-910 (2002).

[6] Galvez, E., "Resource letter spe-1: Single-photon experiments in the undergraduate laboratory," Am. J. Phys. 82, 1018-1028 (2014).

[7] Carlson, J., Olmstead, D., and Beck, M., "Quantum mysteries tested: An experiment implementing hardy's test of local realism," Am. J. Phys. 74, 180-186 (2006).

[8] Guzmán, D., Uribe, L., Valencia, A., Rodríguez, F., and Quiroga, L., "Contrasting classical probability concepts with quantum mechanical behavior in the undergraduate laboratory," Eur. J. Phys. 39, 055039 (2015).

[9] Thorn, J., Neel, M., Donato, V., Bergreen, G., Davies, R., and Beck, M., "Observing the quantum behavior of light in an undergraduate laboratory," Am. J. Phys. 72, 1210-1219 (2004).

[10] Pearson, B. and Jackson, D., "A hands-on introduction to single photons and quantum mechanics for undergraduates," Am. J. Phys. 78, 471-484 (2010).

[11] E. J. Galvez, C. H. Holbrow, M. P., Martin, J., Courtemanche, N., Heilig, L., and Spencer, J., "Interference with correlated photons: Five quantum mechanics experiments for undergraduates," Am. J. Phys. 73, 127-140 (2005).

[12] Galvez, E. and Beck, M., "Quantum optics experiments with single photons for undergraduate laboratories," in [Education and Training in Optics 2007], Nantel, M., ed., 1-8 (2007).

[13] Ashby, J., Schwarz, P., and Schlosshauer, M., "Delayed-choice quantum eraser for the undergraduate laboratory," Am. J. Phys. 84, 95-105 (2016).

[14] Castrillon, J., Galvez, E., Rodriguez, B., and Calderon-Losada, O., "A time-energy delayed-choice interference experiment for the undergraduate laboratory," (in review).

[15] Galvez, E., "Qubit quantum mechanics with correlated-photon experiments," Am. J. Phys. 78, 511-519 (2010).

[16] Carivioto-Lagos, J., P., G. A., Velazquez, V., Lopez-Moreno, E., Grether, M., and Galvez, E., "The hongou-mandel interferometer in the undergraduate laboratory," Eur. J. Phys. 33, 1-8 (2012).

[17] Scott, R., "Alpha instructor manual (unpublished),".

[18] Galvez, E. J., "Photon quantum mechanics: http://departments.colgate.edu/physics/pql.htm."

[19] "Alpha's single photon detector initiative: https://advlab.org/spqm."

[20] Beck, M., "Coincidence counting units (ccus): http://people.reed.edu/ beckm/qm/circuit/circuit.html."

[21] Townsend, J., [Modern Approach to Quantum Mechanics], University Science Books (2012).

[22] Beck, M., [Quantum Mechanics Theory and Experiment], Oxford University Press (2012).

[23] McIntyre, D., Manogue, C., and Tate, J., [Quantum Mechanics: A Paradigms Approach], Addison-Wesley (2012). 